\title{
Physical Activity-Based Interventions Using Electronic Feedback May Be Ineffective in Reducing Pain and Disability in Patients With Chronic Musculoskeletal Pain: A Systematic Review With Meta-Analysis
}

\author{
Crystian B. Oliveira, MSc, ${ }^{a}$ Márcia R. Franco, $\mathrm{PhD}^{a}{ }^{a}$ Chris G. Maher, $\mathrm{PhD},{ }^{b}$ \\ Paulo H. Ferreira, PhD, ${ }^{c}$ Priscila K. Morelhão, MSc, ${ }^{a}$ Tatiana M. Damato, PT, \\ Cynthia Gobbi, MSc, ${ }^{a}$ Rafael Z. Pinto, PhD ${ }^{a, d}$
}

From the ${ }^{a}$ Department of Physical Therapy, Faculty of Science and Technology, Sao Paulo State University, Presidente Prudente, Sao Paulo, Brazil; ' ${ }^{b}$ Sydney School of Public Health, The University of Sydney, Sydney, New South Wales, Australia; ' Musculoskeletal Health Research Group, Faculty of Health Sciences, The University of Sydney, Sydney, New South Wales, Australia; and ${ }^{d}$ Department of Physical Therapy, Federal University of Minas Gerais (UFMG), Belo Horizonte, Minas Gerais, Brazil.

\begin{abstract}
Objective: To investigate the effectiveness of physical activity-based interventions using electronic feedback in reducing pain and disability compared to minimal or no interventions in patients with chronic musculoskeletal pain.

Data Sources: The following electronic databases were searched: EMBASE, MEDLINE, Cochrane Central Register of Controlled Trials, PsycINFO, Cumulative Index to Nursing and Allied Health Literature, SPORTDiscus, Web of Science, Physiotherapy Evidence Database, and main clinical trial registers.

Study Selection: Randomized controlled trials investigating the effect of physical activity interventions using electronic feedback (eg, physical activity monitors) on pain and disability compared to minimal or no interventions in adults with chronic musculoskeletal pain were considered eligible.

Data Extraction: Pooled effects were calculated using the standardized mean difference (SMD), and the Grading of Recommendations Assessment, Development and Evaluation system was used to assess the overall quality of the evidence.

Data Synthesis: Four published randomized controlled trials and 4 registered unpublished randomized controlled trials were included. At shortterm follow-up, pooled estimations showed no significant differences in pain ( 2 trials: $\mathrm{n}=116$; $\mathrm{SMD}=-.50 ; 95 \%$ confidence interval, -1.91 to 0.91 ) and disability ( 2 trials: $\mathrm{n}=116 ; \mathrm{SMD}=-.81 ; 95 \%$ confidence interval, -2.34 to 0.73 ) between physical activity - based interventions and minimal interventions. Similarly, nonsignificant results were found at intermediate-term follow-up. According to Grading of Recommendations Assessment, Development and Evaluation, the overall quality of the evidence was considered to be of low quality.

Conclusions: Our findings suggest that physical activity-based interventions using electronic feedback may be ineffective in reducing pain and disability compared to minimal interventions in patients with chronic musculoskeletal pain. Clinicians should be cautious when implementing this intervention in patients with chronic musculoskeletal pain.
\end{abstract}

Archives of Physical Medicine and Rehabilitation 2018;99:1900-12

(C) 2017 by the American Congress of Rehabilitation Medicine

Supported by São Paulo Research Foundation (FAPESP; grant nos. 2016/03826-5, 2015/

07704-9, 2014/14077-8) and by Capes Foundation, Ministry of Education of Brazil.

Systematic Review Registration No.: CRD42016049730.

Disclosures: none.
In the past decades, chronic musculoskeletal pain has been considered an important health priority. Most chronic pain is caused by musculoskeletal conditions, such as low back pain and 
osteoarthritis. These 2 conditions are the most prevalent chronic musculoskeletal conditions. They pose an important economic burden on individuals and society, ${ }^{1,2}$ and have recently been listed among the leading causes of years lived with disability worldwide. . $^{1,3}$

Physical inactivity is thought to negatively influence the clinical course of chronic musculoskeletal pain conditions. Current evidences suggest that those patients with more severe disability are the ones presenting with low physical activity levels. ${ }^{4,5}$ In terms of prognosis, less physically active or sedentary patients will often report higher pain intensity and disability in the future than will more physically active patients. ${ }^{6,7}$ Furthermore, in a broader sense, physical inactivity may also influence the course of chronic musculoskeletal pain owing to its role in the development of cardiovascular diseases or risk factors for cardiovascular diseases. ${ }^{8}$ There is emerging evidence to suggest that cardiovascular comorbidities (eg, coronary heart disease) or risk factors for cardiovascular diseases (eg, obesity and impaired glucose tolerance) are prevalent in this population ${ }^{9-11}$ and contribute to worse prognosis. ${ }^{12-14}$ Hence, strategies to increase physical activity levels should reasonably be considered when designing treatment for patients with chronic musculoskeletal pain. ${ }^{15}$ Importantly, this is in accordance with health initiatives to promote physical activity in primary care settings. ${ }^{16,17}$

There is evidence showing that physical activity-based interventions are effective in reducing pain and disability at shortterm follow-up in patients with chronic musculoskeletal pain. ${ }^{18,19}$ However, clinical improvements are not sustained for long periods after cessation of treatment. ${ }^{18,19}$ A recently published systematic review $^{20}$ shows that physical activity-based interventions fail to increase physical activity levels in patients with chronic musculoskeletal pain. This finding might be explained by the fact that most physical activity-based interventions in the chronic musculoskeletal field are not specifically designed to change patients' behavior toward an active lifestyle. Nevertheless, we would argue that physical activity-based interventions that incorporate strategies to change physical activity behavior may not only increase physical activity levels but also reduce pain and disability and sustain these improvements at long-term follow-up in patients with chronic musculoskeletal pain.

There are several behavior change strategies to promote physical activity during and after the treatment regimen. ${ }^{21}$ One of these strategies is the provision of feedback ${ }^{22}$ that is considered effective in increasing physical activity ${ }^{23,24}$ and promote healthy habits. ${ }^{25}$ Importantly, current evidence supports the use of feedback to increase adherence to physical activity-based interventions in patients with chronic musculoskeletal pain. ${ }^{26}$ The feedback may include several techniques including self-monitoring, goal setting, action planning, and social comparison that may be delivered through different methods, such as face-to-face or electronic devices. The advantage of electronic devices (ie, web, mobile apps, activity monitors) over the face-to-face mode of delivery is the feature that allows users to set activity goals and self-monitor daily goal-progress, which can be tailored to the needs of the patient

\section{List of abbreviations: \\ CI confidence interval \\ GRADE Grading of Recommendations Assessment, Development and Evaluation \\ PEDro Physiotherapy Evidence Database \\ SMD standardized mean difference}

using real-time information on the biomechanical or physiological consequences of performing physical activity. ${ }^{27}$ In addition, with advances in technology, electronic devices are becoming more affordable for patients. It is unclear, however, whether physical activity-based interventions using electronic feedback can be effective as a treatment for patients with chronic musculoskeletal pain. Therefore, the objective of this systematic review with meta-analysis was to investigate the effectiveness of physical activity-based interventions using electronic feedback in reducing pain and disability compared with no or minimal interventions in patients with chronic musculoskeletal pain. We hypothesized that physical activity-based interventions using electronic feedback would be more effective in reducing pain intensity at short-term follow-up and able to sustain short-term improvements at intermediate and long-term follow-ups.

\section{Methods}

The protocol of this systematic review was previously registered in PROSPERO (CRD42016049730).

The search strategy was performed in the following databases from the earliest record to June 21, 2017: EMBASE, MEDLINE, Cochrane Central Register of Controlled Trials, PsycINFO, Cumulative Index to Nursing and Allied Health Literature, SPORTDiscus, Web of Science, and Physiotherapy Evidence Database (PEDro). We also searched for potential ongoing and unpublished trials by searching the main clinical trial registries: that is, ClinicalTrials.gov, International Standard Randomized Controlled Trial Number Registry, and Australian New Zealand Clinical Trials Registry. The search was also aided by the World Health Organization International Clinical Trials Registry Platform search portal. Citation tracking was performed in relevant publications to identify eligible studies missed by our search. The search strategy was conducted using a combination of search terms, including chronic musculoskeletal pain (eg, low back pain, osteoarthritis, and fibromyalgia), physical activity (eg, walking and exercise), electronic feedback (eg, mobile app, web, or pedometer), and randomized controlled trial. Appendix 1 describes the search strategy used for MEDLINE and Clinicaltrials.gov in this review.

Screening of titles and abstracts was undertaken independently by 2 reviewers (C.B.O. and P.K.M.). After the initial screening, the full texts of potentially eligible articles were assessed following the eligibility criteria by 2 reviewers (C.B.O. and P.K.M.). Any case of disagreement was resolved by consulting a third reviewer (R.Z.P.).

\section{Study selection}

Randomized controlled trials investigating physical activitybased interventions using electronic feedback in patients with chronic musculoskeletal pain were included in this review.

Studies with adults older than 18 years with chronic musculoskeletal pain were included. Chronic musculoskeletal pain was defined as musculoskeletal pain (ie, pain in the axial skeleton or peripheral joints) lasting $>3$ months. Therefore, studies that involved patients with low back pain, neck pain, osteoarthritis, fibromyalgia, and other related conditions were considered eligible. We excluded patients who underwent surgery or had serious or inflammatory rheumatological conditions that require different management strategies, such as tumor, fracture, infection, rheumatoid arthritis, or axial spondyloarthritis. 

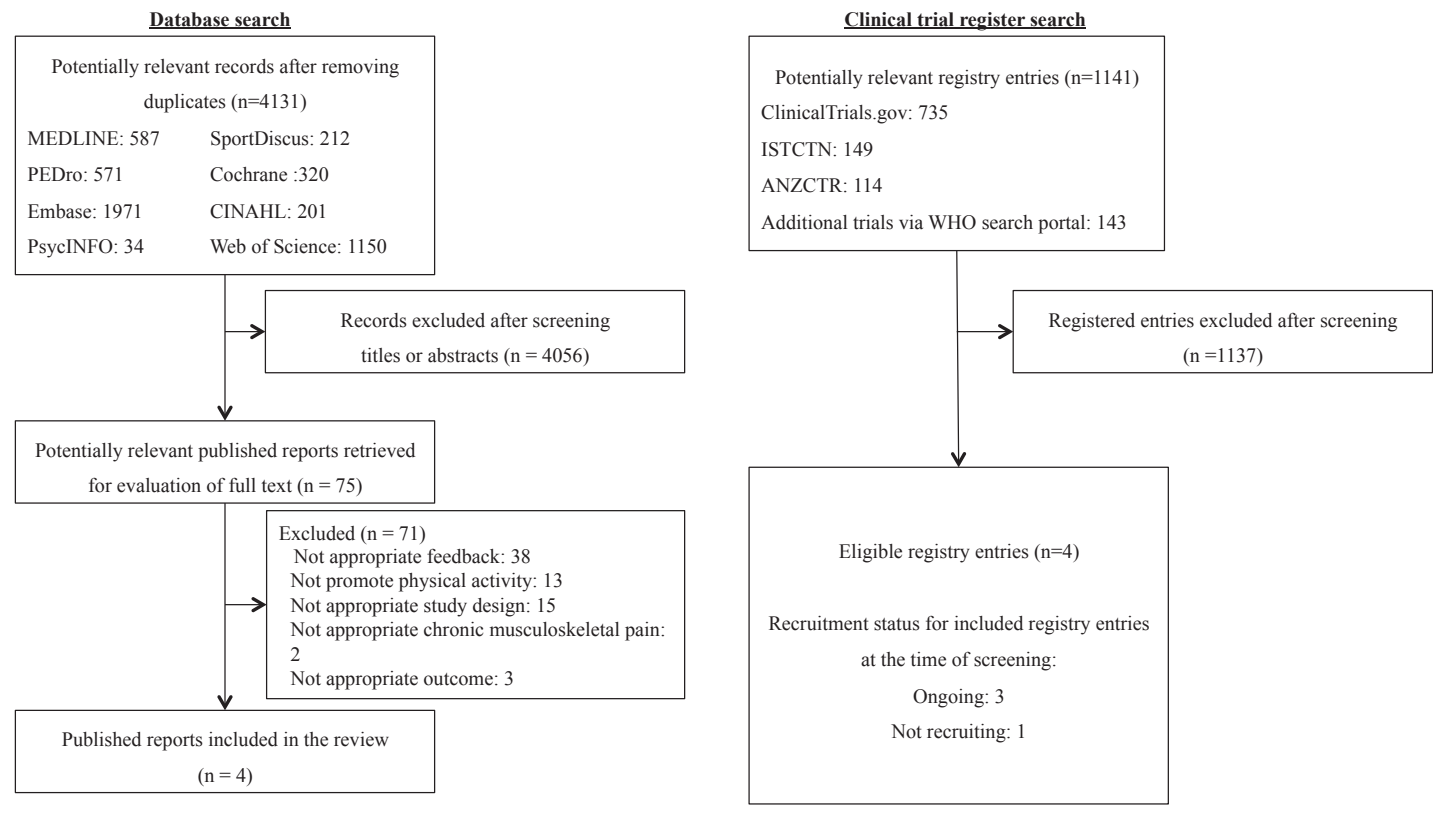

Fig 1 Flow of studies through the review. Abbreviations: ANZCTR, Australian New Zealand Clinical Trials Registry; CINHAL, Cumulative Index to Nursing and Allied Health Literature; ISRCTN, International Standard Randomized Controlled Trial Number; WHO, World Health Organization.

Trials investigating interventions to promote physical activity that included electronic feedback were considered eligible. For this review, we considered the provision of electronic feedback on physical activity behavior following the taxonomy proposed by Michie et al, ${ }^{22}$ which involves providing the participant with data about their own recorded physical activity behavior or commenting on a person's physical activity behavioral performance by setting goals, planning action or facilitating social comparisons. Electronic physical activity feedback was defined as an intervention component delivered via computer (ie, website or e-mail) or smartphone/tablet (ie, mobile app or text messaging) as well as via any wearable device that allows prompt self-monitoring of activity performance, such as pedometers, heart rate monitors, activity monitors, and physical activity trackers (eg, Fitbit, Jawbone, Garmin, Apple watches). Studies investigating exercise-based interventions delivered in a video game format or via web or app technology that do not provide feedback to promote physical activity were excluded. To be considered eligible, the comparator groups needed to be no or minimal interventions including waiting list, placebo, or brief education (eg, provision information on pain management and self-care strategies at 1 session).

In addition, to be considered eligible, studies had to report data for at least 1 relevant primary or secondary outcome. Primary outcomes were pain intensity and disability, whereas secondary outcomes were physical activity level, depression, and quality of life. Depression was not included in the original registered protocol. Nevertheless, we decided to add this outcome as a secondary outcome of this review because of the potential benefits that physical activity-based interventions might have on depression.

\section{Data extraction}

Two independent reviewers (C.B.O and T.M.D.) extracted the data using a standardized form. Any case of disagreement was resolved by consensus. The following data were extracted from the included studies: sample characteristics, intervention group, comparison group, outcome measures, and time-point assessments. Means (final or change values), SDs, and sample sizes were extracted from the included trials. When data were missing, we contacted the authors of the study or estimated data using the recommendations of the Cochrane Handbook for Systematic Reviews of Interventions. If studies reported medians and ranges, means and SDs were calculated. ${ }^{28}$

\section{Risk of bias and quality assessment}

Risk of bias was assessed using the PEDro scale. Two reviewers independently (C.B.O. and C.G.) assessed the risk of bias of included studies. The PEDro scale is considered a reliable ${ }^{29}$ and valid $^{30}$ tool, with 10 items scored as present or absent. If the scores were available in the PEDro, these were extracted to be used in this review.

The overall quality of the evidence was assessed using the Grading of Recommendations Assessment, Development and Evaluation (GRADE) system. ${ }^{31}$ In brief, the GRADE classification was downgraded by 1 level for each of the three domains we considered: (1) study limitations or risk of bias (downgraded when $>25 \%$ of the studies were scored with a high risk of bias; PEDro score, $<6$ ); (2) inconsistency of results (downgraded when substantial heterogeneity was presented with $\mathrm{I}^{2}>50 \%$ ); and (3) imprecision (downgraded if $<400$ participants were included in the meta-analysis). Publication bias was not assessed because of an insufficient number of the included studies. Indirectness was not considered for this review, because this review focused on specific population, comparator, and outcome measures. The quality of the evidence was interpreted as follows: "high quality" (ie, further research is unlikely to change our confidence in the estimate of effect); "moderate quality" (ie, further research is likely to have an important effect on our confidence in the estimate of effect and may change the estimate); "low quality" (ie, further research is likely to have an important effect on our confidence in the estimate of effect and is likely to change the estimate); and "very low quality" (ie, any estimate of effect is uncertain). 
Table 1 Characteristics of the included studies

\begin{tabular}{|c|c|c|c|c|}
\hline Study & $\begin{array}{l}\text { Condition and Patients Characteristics } \\
\text { (Source, Sample Randomized, } \\
\text { and Mean Age) }\end{array}$ & Interventions & Feedback Method & Outcomes and Time Points \\
\hline Kayo et al, $2012^{34}$ & $\begin{array}{l}\text { Condition: Fibromyalgia } \\
\text { Source: Rheumatology clinics } \\
\text { Sample randomized: } 60 \text { patients } \\
\text { (GI: } 30 ; \text { GMI: } 30 \text { ) } \\
\text { Mean age: GI: } 47.7 \pm 5.3 y \text {; } \\
\text { GMI: } 46.1 \pm 6.4 \mathrm{y}\end{array}$ & $\begin{array}{l}\text { GI: Walking in a gymnasium, with each } \\
\text { session including stretching, } \\
\text { conditioning stimulus, and cool down, } \\
\text { and monitored by a heart rate monitor } \\
\text { to increase exercise intensity } \\
\text { GMI: No intervention }\end{array}$ & $\begin{array}{l}\text { Heart rate monitor monitored } \\
\text { during each session }\end{array}$ & $\begin{array}{l}\text { Pain, disability, and quality of life } \\
8-, 16-\text {, and } 28-\text { wk follow-ups }\end{array}$ \\
\hline McDonough et al, $2013^{35}$ & $\begin{array}{l}\text { Condition: Low back pain } \\
\text { Source: Primary care practices } \\
\text { Sample randomized: } 57 \text { patients } \\
\text { (GI: } 40 \text {; GMI: } 17) \\
\text { Mean age (95\% CI): GI: } 48.0 y \\
\quad(43.0-53.0 y) ; \text { GMI: } 51.0 y(42.0 \\
\text {-60.0y) }\end{array}$ & $\begin{array}{l}\text { GI: Graded pedometer-driven walking } \\
\text { program plus an education session } \\
\text { (a single 1-h session) } \\
\text { GMI: Education session } \\
\text { (a single 1-h session) }\end{array}$ & $\begin{array}{l}\text { Pedometer used each day to } \\
\text { self-monitor steps }\end{array}$ & $\begin{array}{l}\text { Pain, disability, and quality of life } \\
\text { 9-wk and 6-mo follow-ups* }\end{array}$ \\
\hline Mengshoel et al, $1992^{36}$ & $\begin{array}{l}\text { Condition: Fibromyalgia } \\
\text { Source: Hospital and general community } \\
\text { Sample randomized: } 35 \text { patients } \\
\text { (GI: 18; GMI: } 17) \\
\text { Median age (range): GI: } 33.5 y(21-42 y) \text {; } \\
\text { GMI: } 34.0 y \text { (25-38y) }\end{array}$ & $\begin{array}{l}\text { GI: Low-effect aerobic dance program } \\
\text { with training intensity prescribed on } \\
\text { the basis of heart rate level } \\
\text { GMI: Instructed to maintain their normal } \\
\text { physical activity levels }\end{array}$ & $\begin{array}{l}\text { Heart rate monitoring controlled } \\
\text { periodically }\end{array}$ & $\begin{array}{l}\text { Pain } \\
\text { 20-wk follow-up }\end{array}$ \\
\hline Sañudo et al, $2015^{37}$ & $\begin{array}{l}\text { Condition: Fibromyalgia } \\
\text { Source: General community } \\
\text { Sample randomized: } 28 \text { patients } \\
\text { (GI: } 16 ; \text { GMI: } 12 \text { ) } \\
\text { Mean age: GI: } 58.0 \pm 2.0 y \text {; } \\
\text { GMI: } 55.0 \pm 2.0 y\end{array}$ & $\begin{array}{l}\text { GI: Exercise sessions included warm-up, } \\
\text { steady-state exercise at } 60 \%-65 \% \text { of } \\
\text { the predicted maximum heart rate, and } \\
15 \text { min of interval training at } 75 \% \\
-80 \% . \text { Exercise intensity was } \\
\text { monitored by a heart rate monitor } \\
\text { GMI: Instructed to maintain normal } \\
\text { activities of daily living }\end{array}$ & Heart rate monitor & $\begin{array}{l}\text { Pain and depression } \\
\text { 24-wk follow-up }\end{array}$ \\
\hline
\end{tabular}




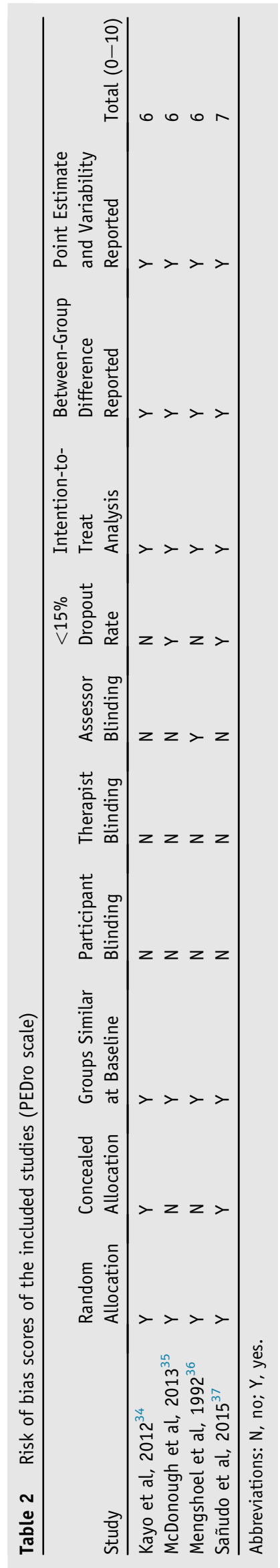

\section{Data analysis}

The outcomes were summarized according to the following time points of assessments: short-term $(<3 \mathrm{mo})$; intermediate-term (between 3 and 12mo); and long-term ( $>12 \mathrm{mo}$ ). If multiple time points fell in the same category, we used the one closest to the end of the treatment, 6 months, and 12 months, respectively. We pooled estimates of the trials using the standardized mean difference (SMD) due to the heterogeneity of the measurement scales for a given outcome. The SMD was interpreted according to Cohen's benchmarks ${ }^{32}$ : small effect size $(\mathrm{SMD}=.20)$; medium effect size $(\mathrm{SMD}=.50)$; and large effect size $(\mathrm{SMD}=.80)$. Heterogeneity between studies was calculated using $\mathrm{I}^{2}$ statistics and by visual inspection of the forest plots. An $\mathrm{I}^{2}$ of $>50 \%$ was considered as substantial heterogeneity. Meta-analyses were calculated using random effects models in RevMan version 5.3. ${ }^{33 \text {, a }}$

\section{Results}

The search strategy yielded 4131 records, and 91 full texts remained to be assessed for eligibility after title and abstract screening. Finally, 4 published reports ${ }^{34-37}$ were included in this review (fig 1). Appendix 2 describes the reasons for exclusion of the full texts. Studies included patients with fibromyalgia $(n=3,75 \%)$ and low back pain $(\mathrm{n}=1,25 \%)$. The sample sizes for included trials ranged from 28 to 60 , and the mean age of participants ranged from 33.5 to 58.0 years. Table 1 summarizes the characteristics of the studies. The physical activity-based interventions were aerobic exercises $(n=3,75 \%)$ and a walking program $(n=1,25 \%)$. The pedometer $(n=1,25 \%)$ and heart rate monitor $(n=3,75 \%)$ were the electronic devices used to provide feedback. The methodological quality of the studies was scored as $6^{34-36}$ or $7^{37}$ out of a maximum score of 10 (table 2). Blinding of patients and therapists was not achieved in the included trials, reflecting the nature of the intervention. One trial $(25 \%)$ reported blinding assessors, and 2 trials $(50 \%)$ reported concealed allocation.

Our search for registered trials retrieved 1379 records (see fig 1). Of these, 4 unpublished trials were considered eligible, recruiting patients with osteoarthritis $(\mathrm{n}=2,50 \%)$ and low back pain $(n=2,50 \%)$. Coaching for physical activity plus activity monitor $(n=3,75 \%)$ and pedometer-based walking program $(\mathrm{n}=1,25 \%)$ were interventions used to promote physical activity, and the pedometer $(n=1,25 \%)$ and Fitbit activity monitor ${ }^{\mathrm{b}}(\mathrm{n}=3$, $75 \%$ ) were the electronic devices used to provide feedback. Appendix 3 gives the details of the unpublished trials.

Figures 2 and 3 show the pooled effects of physical activitybased interventions using electronic feedback on pain intensity and disability compared to minimal interventions. For pain intensity, there is a small, but not significant, effect of physical activity-based interventions compared to minimal interventions at short-term ( 2 trials: $\mathrm{n}=116 ; \mathrm{SMD}=-.50 ; 95 \%$ confidence interval $[\mathrm{CI}],-1.91$ to 0.91 ) and intermediate-term (3 trials: $\mathrm{n}=144 ; \mathrm{SMD}=-.53 ; 95 \% \mathrm{CI},-1.33$ to 0.27 ) follow-ups. For disability, there is a medium, but not significant, effect of physical activity-based interventions compared to minimal interventions at short-term follow-up ( 2 trials: $n=116 ; \mathrm{SMD}=-.81 ; 95 \% \mathrm{CI}$, -2.34 to 0.73 ) and there is a small, but not significant, effect at intermediate follow-up ( 3 trials: $\mathrm{n}=141 ; \mathrm{SMD}=-.67 ; 95 \% \mathrm{CI}$, -1.84 to 0.49 ). The $\mathrm{I}^{2}$ for these meta-analyses ranged from $80 \%$ to $93 \%$, indicating substantial heterogeneity. The overall quality of the evidence was considered low, downgraded for inconsistency and imprecision (table 3 ). 


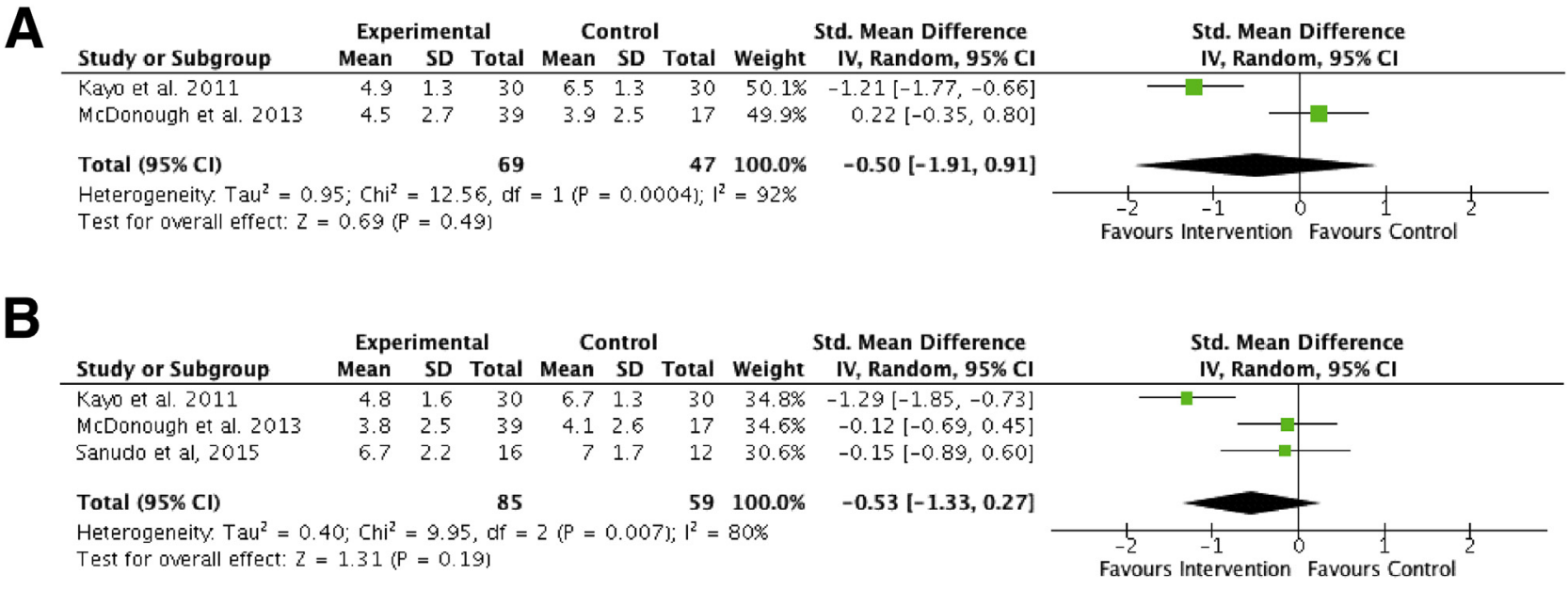

Fig 2 Effect of physical activity-based interventions using electronic feedback on pain intensity at (A) short-term and (B) intermediate-term follow-ups compared to minimal interventions in patients with chronic musculoskeletal pain.

For depression, 1 study $^{37}$ found a nonsignificant effect of the physical activity-based intervention compared to the minimal intervention at intermediate follow-up. For quality of life, 1 study $^{35}$ using a general measure of quality of life found greater improvement favoring the minimal intervention at short-term follow-up but no differences at intermediate follow-up. Another study $^{34}$ reporting the domains of quality of life found significant effects favoring the physical activity-based intervention on bodily pain, vitality, physical functioning, role emotional, and mental health compared to the minimal intervention at intermediate follow-up. None of the studies reported data on physical activity levels.

\section{Discussion}

Our findings revealed that physical activity-based interventions using electronic feedback may not be effective in reducing pain and disability compared to minimal interventions. The nonsignificant results were consistent at all time points of assessments as well as the secondary outcomes (ie, physical activity levels, quality of life, depression). According to GRADE, the evidence for all evaluated outcomes was of low quality, suggesting that further research is likely to change the treatment effects assessed in this review. This is likely to happen in the near future as we identified 4 ongoing trials in clinical trial registries. Nevertheless, on the basis of the results of this review, we would advise clinicians that the use of physical activity-based interventions using electronic devices to provide feedback on physical activity levels should be implemented with caution in routine clinical practice.

The strengths of this review include the use of the GRADE approach to evaluate the overall quality of the evidence and a comprehensive search conducted in 8 electronic databases to locate all relevant published trials in this area. In addition, we performed an extensive search in the major trial registries to identify ongoing studies. The available literature revealed some methodological flaws, such as lack of blinding assessors ${ }^{34,35,37}$ and concealed allocation. ${ }^{35,36}$ A limitation of the current literature was the limited number of trials $(n=4)$ with a relatively small sample size, ranging from 28 to 60 patients. As a result, the overall quality of the evidence for all meta-analyses were downgraded for imprecision because $<400$ patients were included in the analysis. These findings imply that larger studies are warranted to draw more conclusive results.

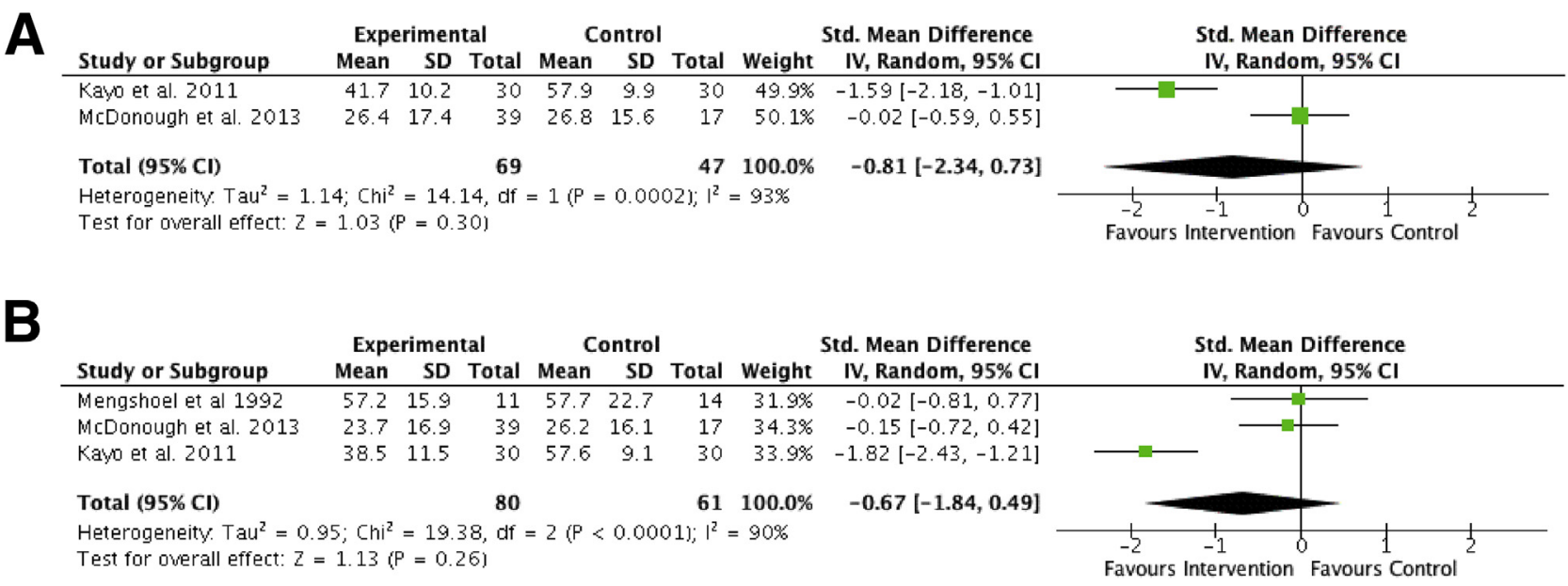

Fig 3 Effect of physical activity-based interventions using electronic feedback compared to minimal interventions on disability at (A) shortterm and (B) intermediate-term follow-ups compared to minimal interventions in patients with chronic musculoskeletal pain. 
Table 3 Evidence table for outcome measures

\begin{tabular}{|c|c|c|c|c|c|c|c|}
\hline \multicolumn{4}{|c|}{ Quality Assessment } & \multicolumn{2}{|c|}{ No. of Patients } & \multirow{2}{*}{$\begin{array}{l}\text { Effect* }^{*} \\
\text { SMD }^{\dagger} \\
(95 \% \mathrm{CI})\end{array}$} & \multirow[b]{2}{*}{$\begin{array}{l}\text { Overall } \\
\text { Quality } \\
\text { of Evidence }\end{array}$} \\
\hline No. of Trials & Risk of Bias & Inconsistency & Imprecision & $\begin{array}{l}\text { Intervention } \\
\text { Group }\end{array}$ & $\begin{array}{l}\text { Minimal } \\
\text { Intervention } \\
\text { Group }\end{array}$ & & \\
\hline \multicolumn{8}{|l|}{ Pain } \\
\hline \multicolumn{8}{|c|}{$\begin{array}{l}\text { Short-term } \\
\text { follow-up }\end{array}$} \\
\hline 2 & $\begin{array}{l}\text { No serious } \\
\text { risk of bias }\end{array}$ & $\begin{array}{l}\text { Serious } \\
\quad \text { inconsistency }\end{array}$ & $\begin{array}{l}\text { Serious } \\
\quad \text { imprecision }\end{array}$ & 69 & 47 & $-0.50(-1.91$ to 0.91$)$ & $\begin{array}{l}\oplus \square \square \\
\text { Low }\end{array}$ \\
\hline \multicolumn{8}{|c|}{$\begin{array}{l}\text { Intermediate } \\
\text { follow-up }\end{array}$} \\
\hline 3 & $\begin{array}{l}\text { No serious } \\
\text { risk of bias }\end{array}$ & $\begin{array}{l}\text { Serious } \\
\quad \text { inconsistency }\end{array}$ & $\begin{array}{l}\text { Serious } \\
\quad \text { imprecision }\end{array}$ & 85 & 59 & $-0.53(-1.33$ to 0.27$)$ & $\begin{array}{l}\oplus \square \square \\
\text { Low }\end{array}$ \\
\hline \multicolumn{8}{|c|}{$\begin{array}{l}\text { Disability } \\
\quad \text { Short-term } \\
\quad \text { follow-up }\end{array}$} \\
\hline 2 & $\begin{array}{l}\text { No serious } \\
\text { risk of bias }\end{array}$ & $\begin{array}{l}\text { Serious } \\
\text { inconsistency }\end{array}$ & $\begin{array}{l}\text { Serious } \\
\quad \text { imprecision }\end{array}$ & 69 & 47 & $-0.81(-2.34$ to 0.73$)$ & $\begin{array}{l}\oplus \square \square \\
\text { Low }\end{array}$ \\
\hline \multicolumn{8}{|c|}{$\begin{array}{l}\text { Intermediate } \\
\text { follow-up }\end{array}$} \\
\hline 3 & $\begin{array}{l}\text { No serious } \\
\text { risk of bias }\end{array}$ & $\begin{array}{l}\text { Serious } \\
\text { inconsistency }\end{array}$ & $\begin{array}{l}\text { Serious } \\
\quad \text { imprecision }\end{array}$ & 80 & 61 & $-0.67(-1.84$ to 0.49$)$ & $\begin{array}{l}\oplus \square \square \\
\text { Low }\end{array}$ \\
\hline $\begin{array}{l}\text { * } \text { Negative } v \\
\dagger \text { The SMD o } \\
\text { More than } \\
\S \text { Wide varia } \\
\text { \& Total num! }\end{array}$ & $\begin{array}{l}\text { avor physical ac } \\
\text { cal activity inte } \\
f \text { participants } \mathrm{fr} \\
\text { point estimates } \\
\text { participants }<30\end{array}$ & $\begin{array}{l}\text { tivity intervention er } \\
\text { rvention enhanced by } \\
\text { om studies with a hi } \\
\text { across studies or if } \\
0 \text { for each outcome. }\end{array}$ & $\begin{array}{l}\text { nhanced by the el } \\
\text { y the electronic } \mathrm{fe} \\
\text { gh risk of bias (PE } \\
\text { heterogeneity bet }\end{array}$ & $\begin{array}{l}\text { ctronic feedbac } \\
\text { edback group as } \\
\text { Dro score, }<6) \text {. } \\
\text { veen trials was }\end{array}$ & $\begin{array}{l}\text { k group. } \\
\text { compared with } \\
\text { arge }\left(\mathrm{I}^{2}>50 \%\right) .\end{array}$ & e minimal intervention 9 & up. \\
\hline
\end{tabular}

To our knowledge, this is the first systematic review investigating the effect of physical activity-based interventions using electronic feedback on clinical outcomes in the chronic musculoskeletal pain field. A recent Cochrane review ${ }^{38}$ found that there is low-quality evidence that physical activity and exercise have small to moderate effects on pain and physical functioning in patients with chronic pain. Our review differs from the previous review because it focuses specifically on the subgroup of physical activity-based interventions that include electronic feedback as part of the intervention, and our results did not support the effectiveness of the intervention in reducing pain and disability. Another recent review $^{39}$ investigating the efficacy of web-based interventions for self-management specifically in patients with chronic low back pain was also unable to demonstrate clinical improvements. This finding together with the results of the present review questions the effectiveness of interventions incorporating electronic feedback in managing patients with chronic musculoskeletal pain.

In the light of the limited evidence on the effectiveness of physical activity-based interventions using electronic feedback, we suggest that clinicians should implement these devices with caution in clinical practice. The financial costs and patients' preferences for electronic devices should be considered in the decision to use these devices in clinical practice. Moreover, a new generation of activity monitors (eg, Fitbit and Jawbone) composed by an interactive interface and additional motivational techniques, such as goal setting, real-time self-monitoring, and social comparison, have emerged. ${ }^{40,41}$ As evidenced by the ongoing trials identified in this review, future studies are likely to contribute and improve the quality of the evidence in this area.

\section{Conclusions}

Physical activity-based interventions using electronic feedback may not be effective in reducing pain and disability in patients with chronic musculoskeletal pain. Future large studies should be conducted with long-term follow-up, incorporating modern activity trackers and additional behavior change strategies to reach definite conclusions about the effectiveness of physical activitybased interventions using behavior change.

\section{Suppliers}

a. RevMan version 5.3; The Cochrane Collaboration.

b. Fitbit; Fitbit, Inc.

\section{Keywords}

Chronic pain; Exercise; Feedback; Physical fitness; Rehabilitation

\section{Corresponding author}

Rafael Z. Pinto, PhD, Department of Physical Therapy, Universidade Federal de Minas Gerais (UFMG), Av Pres Antônio Carlos, 6627, Campus Pampulha, Belo Horizonte, MG 31270-901, Brazil.E-mail address: rafaelzp@ufmg.br. 


\section{Appendix 1 Search strategy for MEDLINE via ovid}

1. exp Back Pain/

2. exp Low Back Pain/

3. exp Sciatica/

4. backache.ab,ti.

5. (lumbar adj pain).ab,ti.

6. coccyx.ab,ti.

7. coccydynia.ab,ti.

8. spondylosis.ab,ti.

9. lumbago.ab,ti.

10. dorsalgia.ab,ti.

11. ((NECK or CERVICAL or KNEE\$ or HIP or HIPS or SHOULDER\$) adj4 PAIN).mp.

12. osteoarthrit*.mp.

13. exp Osteoarthritis/

14. exp Osteoarthritis, Hip/ or exp 0steoarthritis, Spine/ or exp Osteoarthritis, Knee/

15. exp Arthritis, Rheumatoid/

16. ((rheumatoid or reumatoid or reumatoid or rheumatic or reumatic or reumatic or rheumat* or reumat* or reumarthrit*) adj3 (arthrit* or artrit* or diseas* or condition* or nodule*)).tw.

17. exp Fibromyalgia/

18. Fibromyalgia.mp.

19. 1 or 2 or 3 or 4 or 5 or 6 or 7 or 8 or 9 or 10 or 11 or 12 or 13 or 14 or 15 or 16 or 17 or 18

20. exp Exercise/

21. exp Exercise Therapy/

22. $\exp$ Exercise Movement Techniques/

23. exp Motor Activity/

24. Walking/

25. Running/

26. Swimming/

27. Gardening/

28. "physical activit*".mp.

29. exp Sports/

30. exp Recreation/

31. "cardiorespiratory fitness".ab,ti.

32. ((moderate or vigorous $\left.{ }^{*}\right)$ adjactiv $\left.^{*}\right) \cdot$ ab,ti.

33. (("leisure" or "fitness") adj5 ("centre*" or "center*" or "facilit*")).ab,ti.

34. ("exercis*" adj5 ("fit*" or "train*" or "activ*" or "endur*")).ab,ti.

35. ("physical" adj5 ("centre*" or "center*" or "facilit*")).ab,ti.

36. "sport*3 ".ab,ti.

37. "walk*3 ".ab,ti.

38. running.ab,ti.

39. (exercis ${ }^{*} 3$ adj5 aerobic $\left.{ }^{\star}\right) \cdot a b, t i$.

40. "recreation*1".ab,ti.

41. or $/ 20-40$

42. randomized controlled trial.pt.

43. controlled clinical trial.pt.

44. placebo.ab,ti.

45. randomly.ab,ti.

46. trial.ab,ti.

47. groups.ab,ti.

48. "random*".ab,ti.

49. drug therapy.fs.
50. or $/ 42-49$

51. (animals not (humans and animals)).sh.

52. 50 not 51

53. "electronic monitor*".mp.

54. "electronic track*".mp.

55. "electronic activ* monitor*".mp. [mp=title, abstract, original title, name of substance word, subject heading word, keyword heading word, protocol supplementary concept word, rare disease supplementary concept word, unique identifier]

56. "electronic activ* track*".mp.

57. exp Biomedical Technology/

58. "technology based".mp. [mp=title, abstract, original title, name of substance word, subject heading word, keyword heading word, protocol supplementary concept word, rare disease supplementary concept word, unique identifier]

59. "electronic feedback".mp.

60. Coaching.mp.

61. feedback.mp. or exp feedback system/

62. exp monitoring/

63. telemonitoring/

64. telephone/

65. (telerehabilitation or tele-rehabilitation or tele-exercise).mp.

66. pedometer.mp.

67. fitbit.mp.

68. "activity monitor".mp.

69. "step counter".mp.

70. telephone.ab,ti.

71. supervised.mp.

72. (Web or e-mail or computer or Internet or PDA or Website).ab,ti.

73. (number adj2 steps).ab,ti.

74. or $/ 53-73$

75. 19 and 41 and 52 and 75

Data search strategy for ClinicalTrials.gov: ("physical activit*" OR "Motor activity" OR "Sports" OR "Walking") AND ("electronic monitor*" OR "technology based" OR "feedback" OR "activity monitor" OR "telephone" OR "web") 
Appendix 2 Reasons for exclusion of the potentially included full texts

\section{Title of the Study}

The effect of social support features and gamification on a Web-based intervention for rheumatoid arthritis patients: randomized controlled trial

A combined patient and provider intervention for management of osteoarthritis in veterans: a randomized clinical trial

Research to encourage exercise for fibromyalgia (REEF): use of motivational interviewing, outcomes from a randomized controlled trial

Telephone-based self-management of osteoarthritis: a randomized trial

Walking is a feasible physical activity for people with rheumatoid arthritis: a feasibility randomized controlled trial

Telephone coaching to enhance a physiotherapist-prescribed home-based physical activity program for knee osteoarthritis: a randomized clinical trial

The independent and combined effects of intensive weight loss and exercise training on bone mineral density in overweight and obese, older adults with osteoarthritis

Adherence to a Web-based physical activity intervention for patients with knee and/or hip osteoarthritis: a mixed method study

A pilot study of health education via a nurse-run telephone self-management programme for elderly people with osteoarthritis

Coaching patients with early rheumatoid arthritis to healthy physical activity: a multicenter, randomized, controlled study

The implementation of a community-based aerobic walking program for mild to moderate knee osteoarthritis (OA): a knowledge translation (KT) randomized controlled trial (RCT): Part I: The Uptake of the Ottawa Panel clinical practice guidelines (CPGs)

Controlled trial of Internet-based treatment with telephone support for chronic back pain

Randomized trial of the effectiveness of a non-pharmacological multidisciplinary face-to-face treatment program on daily function compared to a telephone-based treatment program in patients with generalized osteoarthritis

Guided internet-delivered acceptance and commitment therapy for chronic pain patients: a randomized controlled trial

Fear of movement/(re)injury in chronic low back pain: education or exposure in vivo as mediator to fear reduction?

Physiotherapy for sleep disturbance in people with chronic low back pain: results of a feasibility randomized controlled trial

Impact of telephone reinforcement and negotiated contracts on behavioral predictors of exercise maintenance in older adults with osteoarthritis

Increasing physical activity in patients with arthritis: a tailored health promotion program

Sedentary behaviour in patients with rheumatoid arthritis: a qualitative study

Progressive resistance training improves overall physical activity levels in patients with early osteoarthritis of the knee: a randomized controlled trial

Efficacy of patient education and supervised exercise vs patient education alone in patients with hip osteoarthritis: a single blind randomized clinical trial

Group-mediated physical activity promotion and mobility in sedentary patients with knee osteoarthritis: results from the IMPACT-pilot trial

Long-term effects of a combined exercise and motivation program in patients with chronic low back pain: a five-year follow-up

Long-term effect of a combined exercise and motivational program on the level of disability of patients with chronic low back pain

Integrating virtual reality with activity management for the treatment of fibromyalgia: acceptability and preliminary efficacy

Medium-/long-term effects of a specific exercise protocol combined with patient education on spine mobility, chronic fatigue, pain, aerobic fitness and level of disability in fibromyalgia

Effectiveness of a cognitive-behavioural group intervention for knee osteoarthritis pain: a randomized controlled trial

A four-week walking exercise programme in patients with knee osteoarthritis improves the ability of dual-task performance: a randomized controlled trial

Impact of the fit and strong intervention on older adults with osteoarthritis

Fit and strong!: bolstering maintenance of physical activity among older adults with lowerextremity osteoarthritis
Main Reason for Exclusion

Does not promote physical activity

Not appropriate feedback

Not appropriate feedback

Not appropriate feedback

Not appropriate feedback

Not appropriate feedback

Not appropriate feedback

Not appropriate outcome

Not appropriate feedback

Not appropriate feedback

Not appropriate study design

Does not promote physical activity Does not promote physical activity

Does not promote physical activity

Not appropriate feedback

Not appropriate study design

Not appropriate feedback

Not appropriate study design Not appropriate study design Not appropriate feedback

Not appropriate feedback

Not appropriate feedback

Not appropriate feedback

Not appropriate feedback

Not appropriate feedback

Not appropriate feedback

Not appropriate feedback

Not appropriate study design

Not appropriate feedback Not appropriate outcome

(continued on next page) 


\section{Appendix 2 (continued)}

Title of the Study

Long-term impact of fit and strong! On older adults with osteoarthritis

Maintenance of physical activity after Internet-based physical activity interventions in patients with rheumatoid arthritis

Supervised walking in comparison with fitness training for chronic back pain in physiotherapy: results of the SWIFT single-blinded randomized controlled trial (ISRCTN17592092)

Telephone coaching can increase activity levels for people with non-chronic low back pain: a randomised trial

Mobile-Web app to self-manage low back pain: randomized controlled trial

Written pain neuroscience education in fibromyalgia: a multicenter randomized controlled trial

Does increasing steps per day predict improvement in physical function and pain interference in adults with fibromyalgia?

First non-contingent respiratory biofeedback placebo versus contingent biofeedback in patients with chronic low back pain: a randomized, controlled, double-blind trial

Effects of spouse-assisted coping skills training and exercise training in patients with osteoarthritic knee pain: a randomized controlled study

The effects of exercise and education, individually or combined, in women with fibromyalgia

Targeting motivation and self-regulation to increase physical activity among patients with rheumatoid arthritis: a randomised controlled trial

Pedometer-based Internet-mediated intervention for adults with chronic low back pain: randomized controlled trial

Explaining physical activity maintenance after a theory-based intervention among patients with rheumatoid arthritis: process evaluation of a randomized controlled trial

Are the benefits of a high-intensity progressive resistance training program sustained in rheumatoid arthritis patients? A 3-year followup study

The Internet-based arthritis self-management program: a one-year randomized trial for patients with arthritis or fibromyalgia

Does moderate-to-high intensity Nordic walking improve functional capacity and pain in fibromyalgia? A prospective randomized controlled trial

Using step cadence goals to increase moderate-to-vigorous intensity physical activity

Effects of self-care education on the health status of inner-city patients with osteoarthritis of the knee

The efficacy of a self-managed Acceptance and Commitment Therapy intervention DVD for physical activity initiation

Type of activity pacing instruction affects physical activity variability in adults with symptomatic knee or hip osteoarthritis

Efficacy of a progressive walking program and glucosamine sulphate supplementation on osteoarthritic symptoms of the hip and knee: a feasibility trial

Physical fitness and work capacity in women with rheumatoid arthritis

Is telephysiotherapy an option for improved quality of life in patients with osteoarthritis of the knee?

Web/Internet-based telemonitoring of a randomized controlled trial evaluating the timeintegrated effects of a 24-week multicomponent intervention on key health outcomes in patients with fibromyalgia

Delivering an Optimised Behavioural Intervention (OBI) to people with low back pain with high psychological risk; results and lessons learnt from a feasibility randomised controlled trial of Contextual Cognitive Behavioural Therapy (CCBT) vs. Physiotherapy

Physical-activity coaching and health status in rheumatoid arthritis: a person-oriented approach

Physical activity coaching of patients with rheumatoid arthritis in everyday practice: a long-term follow-up

Effects of a Web-based intervention for adults with chronic conditions on patient activation: online randomized controlled trial

A randomized controlled trial of a wellness intervention for women with fibromyalgia syndrome

Six-week high-intensity exercise program for middle-aged patients with knee osteoarthritis: a randomized controlled trial

Eight months of physical training in warm water improves physical and mental health in women with fibromyalgia: a randomized controlled trial
Main Reason for Exclusion

Not appropriate feedback

Not appropriate study design

Not appropriate study design

Not appropriate feedback

Does not promote physical activity Does not promote physical activity Not appropriate feedback

Not appropriate feedback

Not appropriate feedback

Not appropriate feedback Not appropriate feedback

Not appropriate study design

Not appropriate feedback

Not appropriate study design

Does not promote physical activity

Not appropriate feedback

Not chronic musculoskeletal pain Does not promote physical activity

Not chronic musculoskeletal pain

Not appropriate feedback

Not appropriate study design

Not appropriate study design Not appropriate feedback

Not appropriate feedback

Not appropriate feedback

Not appropriate feedback Not appropriate feedback

Does not promote physical activity

Does not promote physical activity Not appropriate feedback

Does not promote physical activity

(continued on next page) 
Appendix 2 (continued)

Title of the Study

A brief intervention utilising visual feedback reduces pain and enhances tactile acuity in CLBP patients

Motivational interviewing and exercise programme for community-dwelling older persons with chronic pain: a randomised controlled study

The Web-based osteoarthritis management resource my joint pain improves quality of care: a quasi-experimental study

Engagement and satisfaction with an Internet-based physical activity intervention in patients with rheumatoid arthritis

Using Internet technology to deliver a home-based physical activity intervention for patients with rheumatoid arthritis: a randomized controlled trial

Internet-enhanced management of fibromyalgia: a randomized controlled trial

Effectiveness of a Web-based physical activity intervention in patients with knee and/or hip osteoarthritis: randomized controlled trial

Effects of lifestyle physical activity on perceived symptoms and physical function in adults with fibromyalgia: results of a randomized trial

Effects of lifestyle physical activity in adults with fibromyalgia results at follow-up

A home-based pedometer-driven walking program to increase physical activity in older adults with osteoarthritis of the knee: a preliminary study
Main Reason for Exclusion

Does not promote physical activity

Not appropriate feedback

Not appropriate feedback

Not appropriate outcome

Not appropriate study design

Does not promote physical activity Not appropriate feedback

Not appropriate study design

Not appropriate study design Not appropriate study design 
Appendix 3 Characteristics of the included registry entries or ongoing trials

\begin{tabular}{|c|c|c|c|c|}
\hline $\begin{array}{l}\text { Registration No. } \\
\text { (Registration Year) }\end{array}$ & $\begin{array}{l}\text { Condition, Study Status, }{ }^{*} \\
\text { and Estimated Sample Size }\end{array}$ & Interventions & $\begin{array}{l}\text { Feedback } \\
\text { Method }\end{array}$ & Outcomes and Time Points \\
\hline NCT02284958 (2014) & $\begin{array}{l}\text { Condition: Low back pain } \\
\text { Status: Recruiting } \\
\text { Sample size: } 200\end{array}$ & $\begin{array}{l}\text { GI: Pedometer-based walking program over } 3 \text { mo } \\
\text { with instructions to increase daily step count } \\
\text { plus back care advice and education } \\
\text { GMI: Back care advice and education about self- } \\
\text { management and the benefits of staying active }\end{array}$ & Pedometer & $\begin{array}{l}\text { Disability, physical activity level, and quality of life } \\
\text { 12-wk, 6-mo, and 12-mo follow-ups }\end{array}$ \\
\hline NCT02585323 (2015) & $\begin{array}{l}\text { Condition: Knee osteoarthritis } \\
\text { Status: Recruiting } \\
\text { Sample size: } 60\end{array}$ & $\begin{array}{l}\text { GI: A brief education session on physical } \\
\text { activity, an individual goal-setting session, } \\
\text { and orientation to use the Fitbit and Mac app } \\
\text { GMI: An intervention with } 3 \text { mo of delay, } \\
\text { including a brief education session on physical } \\
\text { activity, an individual goal-setting session, } \\
\text { and orientation to use the Fitbit and Mac app }\end{array}$ & Fitbit & $\begin{array}{l}\text { Pain, disability, physical activity level, and quality of life } \\
3-, 6-\text {, and 9-mo follow-ups }\end{array}$ \\
\hline NCT02315664 (2014) & $\begin{array}{l}\text { Condition: Knee osteoarthritis } \\
\text { Status: Completed } \\
\text { Sample size: } 64\end{array}$ & $\begin{array}{l}\text { GI: A brief education session, remote coaching } \\
\text { with a PT, and use of the Fitbit to } \\
\text { progressively modify the participants' activity } \\
\text { GMI: An intervention with } 2 \text { mo of delay, } \\
\text { including a brief education session on physical } \\
\text { activity, remote coaching with a PT, and use of } \\
\text { the Fitbit to progressively modify the } \\
\text { participants' activity }\end{array}$ & Fitbit & $\begin{array}{l}\text { Pain, disability, physical activity level, and quality of life } \\
2-, 4-\text {, and 6-mo follow-ups }\end{array}$ \\
\hline ACTRN12615000189527 (2015) & $\begin{array}{l}\text { Condition: Low back pain } \\
\text { Status: Recruiting } \\
\text { Sample size: } 68\end{array}$ & $\begin{array}{l}\text { GI: The intervention group will receive an } \\
\text { individually tailored lifestyle modification, } \\
\text { involving } 1 \text { single face-to-face health } \\
\text { coaching session and fortnightly telephone } \\
\text { counseling sessions over } 6 \text { mo. The } \\
\text { intervention will support the use of mobile } \\
\text { health, which includes a specifically designed } \\
\text { mobile web app and physical activity } \\
\text { monitoring device (Fitbit) } \\
\text { GMI: Physical activity booklet advice }\end{array}$ & Fitbit & $\begin{array}{l}\text { Pain, disability, and physical activity level } \\
6 \text { - and 12-mo follow-ups }\end{array}$ \\
\hline
\end{tabular}




\section{References}

1. Cross M, Smith E, Hoy D, et al. The global burden of hip and knee osteoarthritis: estimates from the Global Burden of Disease 2010 study. Ann Rheum Dis 2014;73:1323-30.

2. Hoy D, March L, Brooks P, et al. The global burden of low back pain: estimates from the Global Burden of Disease 2010 study. Ann Rheum Dis 2014;73:968-74.

3. Vos T, Flaxman AD, Naghavi M, et al. Years lived with disability (YLDs) for 1160 sequelae of 289 diseases and injuries 1990-2010: a systematic analysis for the Global Burden of Disease Study 2010. Lancet 2012;380:2163-96.

4. Lin CW, McAuley JH, Macedo L, Barnett DC, Smeets RJ, Verbunt JA. Relationship between physical activity and disability in low back pain: a systematic review and meta-analysis. Pain 2011;152:607-13.

5. Veenhof C, Huisman PA, Barten JA, Takken T, Pisters MF. Factors associated with physical activity in patients with osteoarthritis of the hip or knee: a systematic review. Osteoarthritis Cartilage 2012;20:6-12.

6. Dunlop DD, Song J, Semanik PA, et al. Relation of physical activity time to incident disability in community dwelling adults with or at risk of knee arthritis: prospective cohort study. BMJ 2014;348:g2472.

7. Pinto RZ, Ferreira PH, Kongsted A, Ferreira ML, Maher CG, Kent P. Self-reported moderate-to-vigorous leisure time physical activity predicts less pain and disability over 12 months in chronic and persistent low back pain. Eur J Pain 2014;18:1190-8.

8. Lee IM, Shiroma EJ, Lobelo F, Puska P, Blair SN, Katzmarzyk PT. Effect of physical inactivity on major non-communicable diseases worldwide: an analysis of burden of disease and life expectancy. Lancet 2012;380:219-29.

9. Bliddal H, Leeds AR, Christensen R. Osteoarthritis, obesity and weight loss: evidence, hypotheses and horizons-a scoping review. Obes Rev 2014;15:578-86.

10. Fernandez M, Ordonana JR, Hartvigsen J, et al. Is chronic low back pain associated with the prevalence of coronary heart disease when genetic susceptibility is considered? A co-twin control study of spanish twins. PLoS One 2016;11:e0155194.

11. Ryan CG, McDonough S, Kirwan JP, Leveille S, Martin DJ. An investigation of association between chronic musculoskeletal pain and cardiovascular disease in the Health Survey for England (2008). Eur J Pain 2014;18:740-50.

12. Fernandes GS, Valdes AM. Cardiovascular disease and osteoarthritis: common pathways and patient outcomes. Eur J Clin Invest 2015;45: 405-14.

13. Parsons S, McBeth J, Macfarlane GJ, Hannaford PC, Symmons DP. Self-reported pain severity is associated with a history of coronary heart disease. Eur J Pain 2015;19:167-75.

14. Wilkens P, Scheel IB, Grundnes O, Hellum C, Storheim K. Prognostic factors of prolonged disability in patients with chronic low back pain and lumbar degeneration in primary care: a cohort study. Spine (Phila Pa 1976) 2013:38:65-74.

15. O'Keeffe M, Maher CG, O'Sullivan K. Unlocking the potential of physical activity for back health. 2017;51:760-761.

16. Lobelo F, Stoutenberg M, Hutber A. The Exercise is Medicine Global Health Initiative: a 2014 update. Br J Sports Med 2014;48:1627-33.

17. Parra DC, Bradford ECH, Clark BR, Racette SB, Deusinger SS Population and community-based promotion of physical activity: a priority for physical therapy. Phys Ther 2017;97:159-60.

18. Fransen M, McConnell S, Harmer AR, Van der Esch M, Simic M, Bennell KL. Exercise for osteoarthritis of the knee. Cochrane Database Syst Rev 2015;1:CD004376.

19. van Middelkoop M, Rubinstein SM, Verhagen AP, Ostelo RW, Koes BW, van Tulder MW. Exercise therapy for chronic nonspecific low-back pain. Best Pract Res Clin Rheumatol 2010;24:193-204.

20. Oliveira CB, Franco MR, Maher CG, et al. Physical activity interventions for increasing objectively measured physical activity levels in chronic musculoskeletal pain: systematic review. Arthritis Care Res (Hoboken) 2016;68:1832-42.
21. Bird EL, Baker G, Mutrie N, Ogilvie D, Sahlqvist S, Powell J. Behavior change techniques used to promote walking and cycling: a systematic review. Health Psychol 2013;32:829-38.

22. Michie S, Ashford S, Sniehotta FF, Dombrowski SU, Bishop A, French DP. A refined taxonomy of behaviour change techniques to help people change their physical activity and healthy eating behaviours: the CALO-RE taxonomy. Psychol Health 2011;26:1479-98.

23. Dombrowski SU, Sniehotta FF, Avenell A, Johnston M, MacLennan G, Araújo-Soares V. Identifying active ingredients in complex behavioural interventions for obese adults with obesityrelated co-morbidities or additional risk factors for co-morbidities: a systematic review. Health Psychol Rev 2012;6:7-32.

24. Lewis ZH, Lyons EJ, Jarvis JM, Baillargeon J. Using an electronic activity monitor system as an intervention modality: a systematic review. BMC Public Health 2015;15:585.

25. West R, Evans A, Michie S. Behavior change techniques used in group-based behavioral support by the English stop-smoking services and preliminary assessment of association with short-term quit outcomes. Nicotine Tob Res 2011;13:1316-20.

26. Jordan JL, Holden MA, Mason EE, Foster NE. Interventions to improve adherence to exercise for chronic musculoskeletal pain in adults. Cochrane Database Syst Rev 2010;(1):CD005956.

27. Khaylis A, Yiaslas T, Bergstrom J, Gore-Felton C. A review of efficacious technology-based weight-loss interventions: five key components. Telemed J E Health 2010;16:931-8.

28. Hozo SP, Djulbegovic B, Hozo I. Estimating the mean and variance from the median, range, and the size of a sample. BMC Med Res Methodol 2005;5:13.

29. Maher CG, Sherrington C, Herbert RD, Moseley AM, Elkins M. Reliability of the PEDro scale for rating quality of randomized controlled trials. Phys Ther 2003;83:713-21.

30. Macedo LG, Elkins MR, Maher CG, Moseley AM, Herbert RD, Sherrington C. There was evidence of convergent and construct validity of Physiotherapy Evidence Database quality scale for physiotherapy trials. J Clin Epidemiol 2010;63:920-5.

31. Atkins D, Best D, Briss PA, et al. Grading quality of evidence and strength of recommendations. BMJ 2004;328:1490.

32. Cohen J. Statistical power analysis for the behavioral sciences. 2nd ed. Mahwah: Lawrence Erlbaum Associates; 1988.

33. Review Manager (RevMan) [computer program]. Version 5.3. Copenhagen: The Nordic Cochrane Centre, The Cochrane Collaboration; 2014.

34. Kayo AH, Peccin MS, Sanches CM, Trevisani VF. Effectiveness of physical activity in reducing pain in patients with fibromyalgia: a blinded randomized clinical trial. Rheumatol Int 2012;32:2285-92.

35. McDonough SM, Tully MA, Boyd A, et al. Pedometer-driven walking for chronic low back pain: a feasibility randomized controlled trial. Clin J Pain 2013;29:972-81.

36. Mengshoel AM, Komnaes HB, Forre O. The effects of 20 weeks of physical fitness training in female patients with fibromyalgia. Clin Exp Rheumatol 1992;10:345-9.

37. Sañudo B, Carrasco L, Hoyo M, Figueroa A, Saxton JM. Vagal modulation and symptomatology following a 6-month aerobic exercise program for women with fibromyalgia. Clin Exp Rheumatol 2015;33(1 Suppl 88):S41-5.

38. Geneen LJ, Moore RA, Clarke C, Martin D, Colvin LA, Smith BH. Physical activity and exercise for chronic pain in adults: an overview of Cochrane Reviews. Cochrane Database Syst Rev 2017;1:CD011279.

39. Garg S, Garg D, Turin TC, Chowdhury MFU. Web-based interventions for chronic back pain: a systematic review. J Med Internet Res 2016;18:e139.

40. Evenson KR, Goto MM, Furberg RD. Systematic review of the validity and reliability of consumer-wearable activity trackers. Int J Behav Nutr Phys Act 2015;12:159.

41. Lyons EJ, Lewis ZH, Mayrsohn BG, Rowland JL. Behavior change techniques implemented in electronic lifestyle activity monitors: a systematic content analysis. J Med Internet Res 2014;16:e192. 